\title{
Radiosurgery for Brain Metastases: Changing Practice Patterns and Disparities in the United States
}

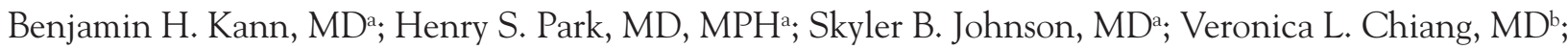 \\ and James B. Yu, MD, MHS ${ }^{a}$
}

\begin{abstract}
Background: Management of brain metastases typically includes radiotherapy (RT) with conventional fractionation and/or stereotactic radiosurgery (SRS). However, optimal indications and practice patterns for SRS remain unclear. We sought to evaluate national practice patterns for patients with metastatic disease receiving brain RT. Methods: We queried the National Cancer Data Base (NCDB) for patients diagnosed with metastatic non-small cell lung cancer, breast cancer, colorectal cancer, or melanoma from 2004 to 2014 who received upfront brain RT. Patients were divided into SRS and non-SRS cohorts. Patient and facility-level SRS predictors were analyzed with chi-square tests and logistic regression, and uptake trends were approximated with linear regression. Survival by diagnosis year was analyzed with the Kaplan-Meier method. Results: Of 75,953 patients, 12,250 (16.1\%) received SRS and 63,703 (83.9\%) received non-SRS. From 2004 to 2014 , the proportion of patients receiving SRS annually increased (from $9.8 \%$ to $25.6 \% ; P<.001$ ), and the proportion of facilities using SRS annually increased (from $31.2 \%$ to $50.4 \% ; P<.001$ ). On multivariable analysis, nonwhite race, nonprivate insurance, and residence in lowerincome or less-educated regions predicted lower SRS use ( $P<.05$ for each). During the study period, SRS use increased disproportionally among patients with private insurance or who resided in higher-income or higher-educated regions. From 2004 to 2013, 1-year actuarial survival improved from $24.1 \%$ to $49.6 \%$ for patients selected for SRS and from $21.0 \%$ to $26.3 \%$ for non-SRS patients $(P<.001)$. Conclusions: This NCDB analysis demonstrates steadily increasing-although modest overall-brain SRS use for patients with metastatic disease in the United States and identifies several progressively widening sociodemographic disparities in the adoption of SRS. Further research is needed to determine the reasons for these worsening disparities and their clinical implications on intracranial control, neurocognitive toxicities, quality of life, and survival for patients with brain metastases.
\end{abstract}

More than $20 \%$ of patients with cancer will develop brain metastases. ${ }^{1}$ Primary malignancies most commonly associated with brain metastases are lung, breast, and gastrointestinal cancers and melanoma, constituting up to $80 \%$ of brain metastases. ${ }^{1-3}$ Management of brain metastases often consists of tumor-directed treatment with radiotherapy (RT). Traditionally, RT had been administered with conventionally fractionated whole-brain RT (WBRT), although over the past several decades, stereotactic radiosurgery (SRS), targeted at individual cranial lesions, has become accepted. ${ }^{4-6} \mathrm{Sev}$ eral randomized trials demonstrated equivalent survival

From the Departments of aTherapeutic Radiology and ${ }^{b}$ Neurosurgery, Yale University School of Medicine, New Haven, Connecticut.

Submitted May 23, 2017; accepted for publication July 5, 2017.

The authors have disclosed that they have no financial interests, with upfront SRS and WBRT for patients with 1 to 3 brain metastases. ${ }^{7,8}$ In the context of these trials, there was a modest national increase in SRS use observed in Medicare patients with metastatic non-small cell lung cancer (NSCLC) from 2000 to 2005. ${ }^{9}$ Since then, several randomized trials showed that SRS upfront without WBRT did not compromise survival and was associated with fewer adverse neurocognitive effects. ${ }^{10-12}$ SRS use may also vary by primary disease site. ${ }^{13,14}$ Additionally, cost concerns and access to facilities with SRS programs may vary and introduce disparities for certain patient groups. arrangements, affiliations, or commercial interests with the manufacturers of any products discussed in this article or their competitors.

Correspondence: Benjamin H. Kann, MD, Yale Therapeutic Radiology, Yale University School of Medicine, 35 Park Street, LL509, New Haven, CT 06519. E-mail: Benjamin.kann@yale.edu 
Utilization of SRS may vary widely on a national scale. ${ }^{15,16}$ Therefore, we sought to evaluate recent national SRS treatment patterns, institutional adoption, and disparities across 4 of the malignancies most associated with brain metastases in the United States.

\section{Methods}

\section{Data Source and Study Population}

We conducted a retrospective analysis of the $\mathrm{Na}$ tional Cancer Data Base (NCDB), a joint project of the Commission on Cancer ( $\mathrm{CoC}$ ) of the American College of Surgeons and the American Cancer Society. It is a hospital-based database that contains deidentified information from approximately 70\% of newly diagnosed cancers in the United States. The NCDB contains information that is unavailable in the SEER database, including treatment details pertaining to RT dose, technique, and target. Treatments coded in the NCDB capture a patient's firstcourse treatment for the corresponding diagnosis. The data used in this study are derived from deidentified NCDB participant user files. The American College of Surgeons and the CoC have not verified and are not responsible for the analytical or statistical methodology used, nor the conclusions drawn from these data by the investigators.

We pooled the NCDB participant user files for NSCLC, breast cancer, colorectal cancer (CRC), and melanoma. We identified patients aged $\geq 18$ years, diagnosed with histology- or cytology-confirmed NSCLC, breast cancer, CRC, or melanoma between 2004 and 2014, with metastatic disease, who were treated with radiation to the brain. Patients with unknown radiation modality and dose were excluded. Radiation treatment modality is explicitly coded in the NCDB, and patients were divided into 2 cohorts: SRS or non-SRS. The SRS cohort was defined as patients who received "stereotactic radiosurgery, NOS [not otherwise specified]," "LINAC radiosurgery," or "gamma knife radiosurgery," or received external-beam RT with fraction size $\geq 6$ Gy. The non-SRS cohort included all other patients receiving external-beam RT to the brain who met the inclusion criteria.

\section{Statistical Methodology}

Patient-level characteristics included age, year of diagnosis, sex, malignancy type, Charlson-Deyo comorbidity score (CDS), race/ethnicity, insurance status, distance from facility, median household income in patient's zip code, residence in a metropolitan area, and educational status in the patient's zip code (percentage of adults who did not graduate high school). Income and educational status in the NCDB were derived from the 2012 American Community Survey data. ${ }^{17}$ Facility-level characteristics included region and academic affiliation. Data on the number of brain metastases are not available in the NCDB.

Demographic, clinical, and temporal associations were evaluated between patients in the SRS and non-SRS cohorts using graphical assessment, chi-square, and Wilcoxon rank sum tests. Univariable (UVA) multilevel, mixed-effects regression was performed, accounting for the reporting facility as a random effect, and odds ratios (ORs) were calculated. Multivariable (MVA) multilevel, mixed-effects logistic regression, again accounting for the reporting facility, was conducted using all variables with a significant association with SRS on UVA; adjusted ORs (aORs) were calculated. Variables were tested for collinearity and interaction. Sensitivity analysis with MVA logistic regression was also conducted after excluding patients with melanoma, given the predominantly white population reflected by this disease type.

For variables that were significant predictors of SRS use, analysis of temporal trends was conducted to assess for differential uptake across variables over the study period with graphical assessment. Uptake slopes $(\beta)$ were approximated with linear regression and compared among categories with Wald tests. ${ }^{18,19}$

A facility-level analysis was conducted to determine the rates of SRS adoption by US facility. For this analysis, only patients who received at least part of their first treatment course at the reporting facility were included. Facilities that reported at least 1 case of SRS during a given year were classified as an SRS facility for that year. Facilities that did not report a single case of SRS were classified as a non-SRS facility for that year.

A survival analysis was conducted after excluding patients without documented follow-up. Followup was documented in our NCDB user file through 2013. Survival was defined as time from diagnosis to death or last follow-up. One-year actuarial survival was analyzed annually during the study period using 
Kann et al

the Kaplan-Meier method, and cohorts were compared with log-rank tests.

All tests were 2 -sided, and $P<.05$ was considered statistically significant. Analyses were performed using Stata/SE 13 (StataCorp, College Station, TX).

\section{Results}

\section{Patient Characteristics and Overall Trends of SRS Use}

We identified 75,953 patients who met our inclusion criteria for analysis (see supplemental eFigure $\underline{1 \text {, available online with this article at JNCCN.org). }}$ Of these, $12,250(16.1 \%)$ were in the SRS cohort and $63,703(83.9 \%)$ were in the non-SRS cohort. Of 68,710 patients with NSCLC, 10,799 (15.7\%) received SRS; of 3,565 patients with melanoma, 952 (26.7\%) received SRS; of 2,909 patients with breast cancer, $348(12.0 \%)$ received SRS; and of 769 patients with CRC, 151 (19.6\%) received SRS (Table $1)$. The overall utilization rate for SRS increased from $9.8 \%$ in 2004 to $25.6 \%$ in $2014(P<.001)$, with an average annual increase of $1.6 \%$ per year (Figure $1)$. The annual increase of SRS uptake was higher from 2009 to 2014 than from 2004 to 2009 (2.6\% vs $0.5 \%$ per year, respectively).

\section{Predictors of SRS Use}

Predictors of SRS use on UVA and MVA with ORs and confidence intervals are shown in Table 2. On MVA, predictors of higher SRS use were later year of diagnosis; melanoma, CRC, or NSCLC compared with breast cancer; private insurance, Medicare, or Medicaid compared with no insurance; residence in zip code with a higher rate of high school education; and greater distance from facility. Predictors of lower SRS use were black race, Hispanic ethnicity, CDS 2, residence in zip code with lower median household income, and nonacademic reporting facility. MVA excluding patients with melanoma demonstrated similar results (data not shown).

\section{SRS Uptake in Clinical and Sociodemographic Subgroups}

SRS use increased across all disease sites during the study period (supplemental eFigure 2). From 2004 to 2014 , the proportion of patients receiving SRS increased from $9.8 \%$ to $25.0 \%$ for NSCLC, $7.9 \%$ to $18.8 \%$ for breast cancer, $4.8 \%$ to $40.6 \%$ for CRC, and $12.7 \%$ to $36.2 \%$ for melanoma. According to linear regression approximation, SRS increased most for patients with melanoma $(\beta=2.13)$ compared with those with NSCLC $(\beta=1.28 ; P<.001)$ and breast cancer $(\beta=1.02 ; P<.001)$, although was similar to uptake in patients with $\mathrm{CRC}(\beta=2.09 ; P=.96)$. SRS use increased more for patients with income $\geq \$ 63,000$ than for those with lower income $(\beta=1.86$ vs $1.13 ; P<.001$; Figure $2 \mathrm{~A})$; more for patients with private insurance $(\beta=1.64)$ than those with Medicare $(\beta=1.29 ; P=.001)$, Medicaid $(\beta=.99 ; P=.06$ [relative to Medicare]), or no insurance $(\beta=.74 ; P=.01$ [relative to Medicaid]) (Figure $2 \mathrm{~B}$ ). SRS use also increased more for patients treated at academic versus nonacademic facilities $(\beta=2.26$ vs $1.01 ; P<.001)$ (Figure $2 \mathrm{C}$ ); for patients in regions with a greater level of high school education $(\beta=1.48$ vs 1.10 ; $P<.001$ ) (Figure 2D); and among Hispanic versus black patients $(\beta=1.69$ vs $1.28 ; P=.03)$, but not more than among white patients $(\beta=1.34 ; P=.06)$ (supplemental eFigure 3).

\section{Facility-Level Analysis of SRS Uptake}

Among the study population, 73,077 patients $(96.2 \%)$ received at least part of their treatment at the reporting facility, with a total of 1,207 treatment facilities reporting cases from 2004 to 2014. Not all facilities reported cases every year, and the number of facilities reporting yearly ranged from a low of 913 to a high of 1,044 . The number of SRS facilities increased yearly from $285(31.2 \%)$ in 2004 to 515 (50.4\%) in 2014 (Figure 3).

\section{Survival Trends Analysis}

Among the 67,448 patients in the survival analysis, 1 -year actuarial overall survival was $26.6 \%$ for the entire population; 1 -year survival was $40.9 \%$ for SRS patients and $24.1 \%$ for non-SRS patients $(P<.001)$. One-year survival increased for patients with each year of diagnosis, from $21.3 \%$ in 2004 (95\% CI, $20.2 \%-22.4 \%)$ to $31.5 \%$ in 2013 (95\% CI, 30.6\%$32.6 \%$ ) (Figure 4). From 2004 to 2013, 1-year survival increased from $24.1 \%$ (95\% CI, 20.6\%-28.3\%) to $49.6 \%$ (95\% CI, $47.4 \%-52.0 \%$ ) among SRS patients, and from $21.0 \%$ (95\% CI, 19.8\%-22.2\%) to $26.3 \%(95 \% \mathrm{CI}, 25.3 \%-27.5 \%)$ in non-SRS patients. 
Radiosurgery for Brain Metastases

\begin{tabular}{|c|c|c|c|c|c|c|}
\hline Variable & $\begin{array}{c}\text { Total, } \mathrm{n} \\
(\mathrm{N}=75,953)\end{array}$ & $\begin{array}{c}\text { SRS, } n \\
(\mathrm{~N}=12,250)\end{array}$ & $\%$ & $\begin{array}{l}\text { Non-SRS, } n \\
(\mathrm{~N}=63,703)\end{array}$ & $\%$ & $P$ Value \\
\hline Median age, y & 64 & 64 & & 63 & & $<.001$ \\
\hline Range & $18-90$ & $19-90$ & & $18-90$ & & \\
\hline Year of diagnosis & & & & & & $<.001$ \\
\hline 2004-2007 & 21,803 & 2,537 & 11.6 & 19,266 & 88.4 & \\
\hline $2008-2011$ & 29,061 & 4,187 & 14.4 & 24,874 & 85.6 & \\
\hline $2012-2014$ & 25,089 & 5,526 & 22.0 & 19,563 & 78.0 & \\
\hline Cancer site & & & & & & $<.001$ \\
\hline NSCLC & 68,710 & 10,799 & 15.7 & 57,911 & 84.3 & \\
\hline Melanoma & 3,565 & 952 & 26.7 & 2,613 & 73.3 & \\
\hline Breast & 2,909 & 348 & 12.0 & 2,561 & 88.0 & \\
\hline Colorectal & 769 & 151 & 19.6 & 618 & 80.4 & \\
\hline Sex & & & & & & .008 \\
\hline Male & 39,085 & 6,169 & 15.8 & 32,916 & 84.2 & \\
\hline Female & 36,868 & 6,081 & 16.5 & 30,787 & 83.5 & \\
\hline Race/Ethnicity & & & & & & $<.001$ \\
\hline White, non-Hispanic & 57,542 & 9,639 & 16.8 & 47,903 & 83.3 & \\
\hline White, Hispanic & 2,028 & 274 & 13.5 & 1,754 & 86.5 & \\
\hline Black & 9,383 & 1,324 & 14.1 & 8,059 & 85.9 & \\
\hline Other/NOS & 7,000 & 1,013 & 14.5 & 5,987 & 85.5 & \\
\hline Charlson-Deyo comorbidity score & & & & & & $<.001$ \\
\hline 0 & 51,627 & 8,561 & 16.6 & 43,066 & 83.4 & \\
\hline 1 & 17,134 & 2,666 & 15.6 & 14,468 & 84.4 & \\
\hline 2 & 7,192 & 1,023 & 14.2 & 6,169 & 85.8 & \\
\hline Insurance status & & & & & & $<.001$ \\
\hline None & 4,480 & 424 & 9.5 & 4,056 & 90.5 & \\
\hline Medicaid & 7,527 & 993 & 13.2 & 6,534 & 86.8 & \\
\hline Medicare $^{\mathrm{a}}$ & 34,435 & 5,779 & 16.8 & 28,656 & 83.22 & \\
\hline Unknown & 1,371 & 225 & 16.4 & 1,146 & 83.6 & \\
\hline Income & & & & & & $<.001$ \\
\hline$\geq \$ 63,000$ USD & 20,621 & 4,011 & 19.5 & 16,610 & 80.6 & \\
\hline$<\$ 63,000$ USD & 53,652 & 8,018 & 14.9 & 45,634 & 85.1 & \\
\hline Unknown & 1,680 & 221 & 13.2 & 1,459 & 86.8 & \\
\hline No high school education & & & & & & $<.001$ \\
\hline$\geq 13 \%$ & 29,220 & 4,057 & 13.9 & 25,163 & 86.1 & \\
\hline$<13 \%$ & 45,079 & 7,973 & 17.7 & 37,106 & 82.3 & \\
\hline Unknown & 1,654 & 220 & 13.3 & 1,434 & 86.7 & \\
\hline Facility affiliation & & & & & & $<.001$ \\
\hline Academic & 26,264 & 5,741 & 21.9 & 20,523 & 78.1 & \\
\hline Nonacademic & 48,551 & 6,293 & 13.0 & 42,258 & 87.0 & \\
\hline Unknown & 1,138 & 216 & 19.0 & 922 & 81.0 & \\
\hline Facility region & & & & & & $<.001$ \\
\hline Northeast & 16,151 & 2,918 & 18.1 & 13,233 & 81.9 & \\
\hline South & 21,390 & 3,431 & 16.0 & 17,959 & 84.0 & \\
\hline Midwest & 22,404 & 3,304 & 14.8 & 19,100 & 85.2 & \\
\hline West & 14,870 & 2,381 & 16.0 & 12,489 & 84.0 & \\
\hline Unknown & 1,138 & 216 & 19.0 & 922 & 81.0 & \\
\hline Distance from facility & & & & & & $<.001$ \\
\hline$<20$ miles & 53,597 & 7,777 & 14.5 & 45,820 & 85.5 & \\
\hline$\geq 20$ miles & 22,356 & 4,473 & 20.0 & 17,883 & 80.0 & \\
\hline Region & & & & & & $<.001$ \\
\hline Metropolitan area & 58,105 & 9,685 & 16.7 & 48,420 & 83.3 & \\
\hline Nonmetropolitan area & 14,865 & 2,072 & 14.0 & 12,774 & 86.0 & \\
\hline Unknown & 3,002 & 493 & 16.4 & 2,509 & 83.6 & \\
\hline
\end{tabular}

Abbreviations: NOS, not otherwise specified; NSCLC, non-small cell lung cancer; SRS, stereotactic radiosurgery. ${ }^{a}$ Medicare group includes Medicare and other government insurance. 


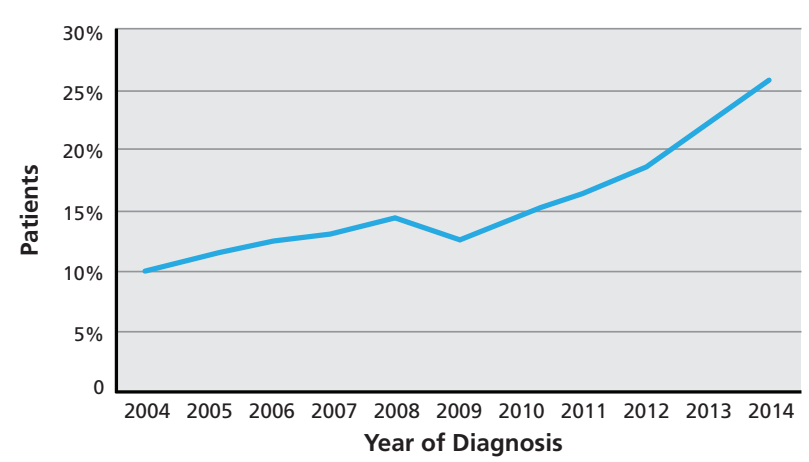

Figure 1. Brain stereotactic radiosurgery for patients with metastatic non-small cell lung cancer, breast cancer, colorectal cancer, or melanoma in the United States (2004-2014).

\section{Discussion}

In this large national database analysis of 4 of the malignancies most commonly associated with brain metastases, overall use and adoption of upfront SRS for RT of brain metastases increased steadily from
2004 to 2014, although remaining modest overall. In the most recent study year, $50 \%$ of facilities did not report using brain SRS, demonstrating potential access barriers to SRS use. The study further revealed socioeconomic and racial/ethnic disparities in the use and uptake of SRS. Finally, findings showed improvement in 1-year survival among patients with metastatic cancer receiving brain RT over the course of the study period. This improvement was seen predominantly in patients selected to receive SRS, although causality cannot be determined.

This study provides an overview of the use and adoption of brain SRS in the modern era of RT for brain metastases, serves as a foundation for further research into drivers of uptake and disparities, and highlights the need for prospective comparative effectiveness research in this area.

To our knowledge, no prior study has investigated the use and uptake of brain SRS across multiple disease sites and age groups. Our findings expand on prior study of SRS use for NSCLC brain metastases

\begin{tabular}{|c|c|c|c|c|c|c|}
\hline \multirow[b]{2}{*}{ Variable (ref) } & \multicolumn{3}{|c|}{ Univariable Analysis } & \multicolumn{3}{|c|}{ Multivariable Analysis } \\
\hline & OR & $P$ Value & $95 \% \mathrm{Cl}$ & aORs & $P$ Value & $95 \% \mathrm{Cl}$ \\
\hline Age, y (continuous) & 1.01 & $<.001$ & $1.01-1.01$ & 1.01 & $<.001$ & $1.00-1.01$ \\
\hline Year of diagnosis (continuous) & 1.12 & $<.001$ & $1.11-1.13$ & 1.12 & $<.001$ & $1.11-1.12$ \\
\hline \multicolumn{7}{|l|}{ Cancer site (NSCLC) } \\
\hline Breast & 0.72 & $<.001$ & $0.63-0.82$ & 0.74 & $<.001$ & $0.65-0.84$ \\
\hline Colorectal & 1.43 & .001 & $1.17-1.76$ & 1.35 & .004 & $1.10-1.66$ \\
\hline Melanoma & 1.80 & $<.001$ & $1.65-1.97$ & 1.64 & $<.001$ & $1.49-1.80$ \\
\hline \multicolumn{7}{|l|}{ Sex (male) } \\
\hline Female & 1.03 & .25 & $0.98-1.07$ & - & - & - \\
\hline \multicolumn{7}{|l|}{ Race/Ethnicity (white) } \\
\hline Black & 0.71 & $<.001$ & $0.66-0.76$ & 0.88 & .001 & $0.81-0.95$ \\
\hline White Hispanic & 0.72 & $<.001$ & $0.62-0.83$ & 0.85 & .045 & $0.73-0.99$ \\
\hline Other/NOS & 0.79 & $<.001$ & $0.73-0.86$ & 0.92 & .049 & $0.84-0.99$ \\
\hline \multicolumn{7}{|c|}{ Charlson-Deyo comorbidity score (0) } \\
\hline 1 & 0.98 & .50 & $0.93-1.04$ & 0.97 & .26 & $0.92-1.02$ \\
\hline 2 & 0.86 & $<.001$ & $0.80-0.93$ & 0.84 & $<.001$ & $0.77-0.91$ \\
\hline \multicolumn{7}{|l|}{ Insurance status (none) } \\
\hline Private & 1.86 & $<.001$ & $1.65-2.09$ & 1.77 & $<.001$ & $1.57-1.99$ \\
\hline Medicaid & 1.37 & $<.001$ & $1.19-1.56$ & 1.34 & $<.001$ & $1.17-1.54$ \\
\hline Medicare $^{a}$ & 1.96 & $<.001$ & $1.74-2.20$ & 1.71 & $<.001$ & $1.51-1.93$ \\
\hline \multicolumn{7}{|l|}{ Median income ( $\geq \$ 63,000$ USD) } \\
\hline$<\$ 63,000$ USD & 0.80 & $<.001$ & $0.76-0.85$ & 0.90 & $<.001$ & $0.84-0.95$ \\
\hline \multicolumn{7}{|l|}{ No high school education $(\geq 13 \%)$} \\
\hline$<13 \%$ & 1.28 & $<.001$ & $1.22-1.35$ & 1.18 & $<.001$ & $1.12-1.25$ \\
\hline \multicolumn{7}{|l|}{ Facility affiliation (academic) } \\
\hline Nonacademic & 0.50 & $<.001$ & $0.43-0.58$ & 0.52 & $<.001$ & $0.44-0.60$ \\
\hline \multicolumn{7}{|l|}{ Distance to facility (<20 miles) } \\
\hline$\geq 20$ miles & 1.39 & $<.001$ & $1.32-1.46$ & 1.36 & $<.001$ & $1.29-1.44$ \\
\hline \multicolumn{7}{|l|}{ Facility region (Northeast) } \\
\hline South & 0.85 & .13 & $0.69-1.05$ & - & - & - \\
\hline Midwest & 0.84 & .09 & $0.69-1.03$ & - & - & - \\
\hline West & 1.04 & .69 & $0.85-1.29$ & - & - & - \\
\hline \multicolumn{7}{|c|}{ City environment (metropolitan area) } \\
\hline Nonmetropolitan area & 1.05 & .16 & $0.98-1.12$ & - & - & - \\
\hline
\end{tabular}

Abbreviations: aORs, adjusted odds ratios; NOS, not otherwise specified; NSCLC, non-small cell lung cancer; OR, odds ratio.

aMedicare subgroup includes patients with Medicare or "other government" insurance. 
A

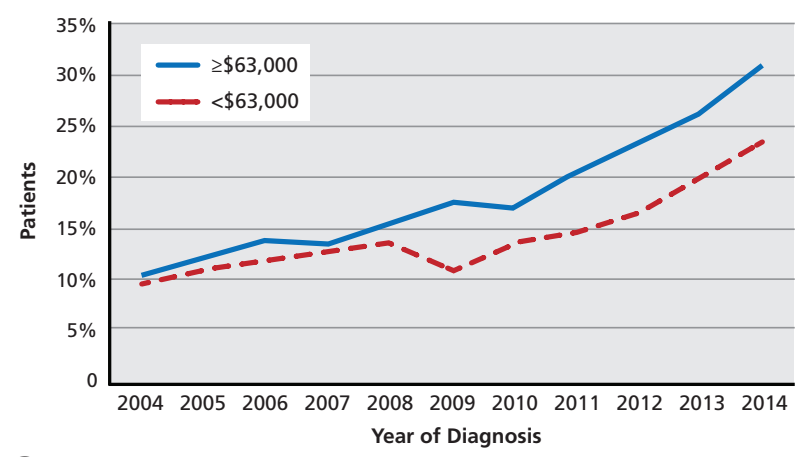

C

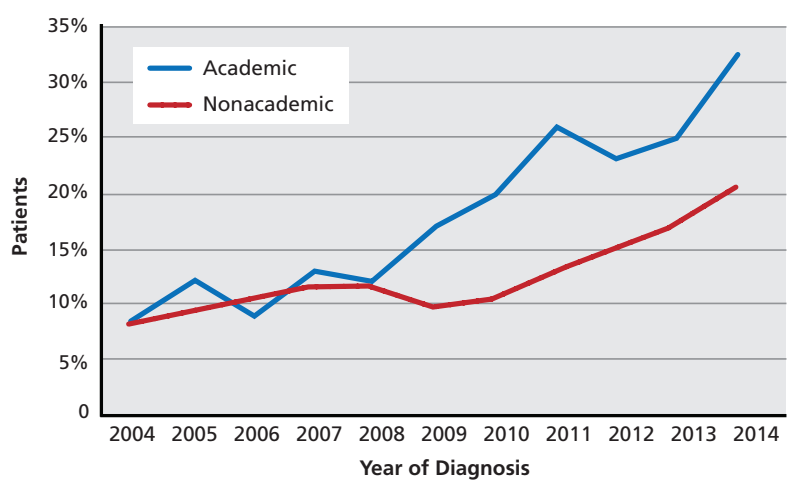

B

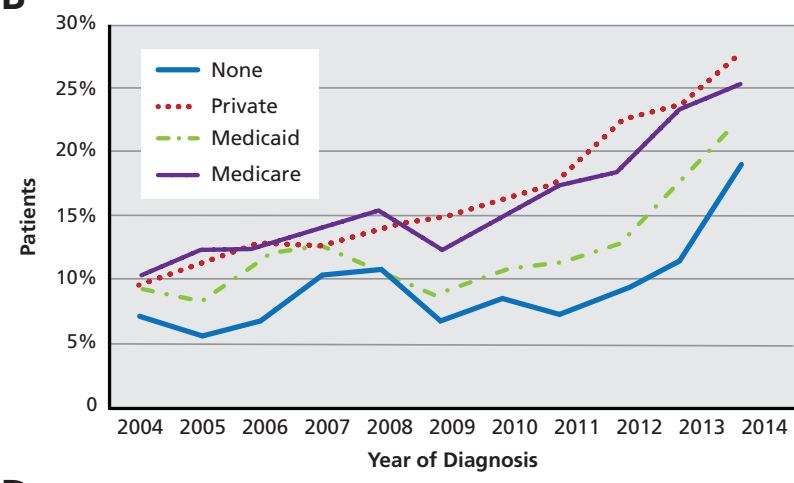

D

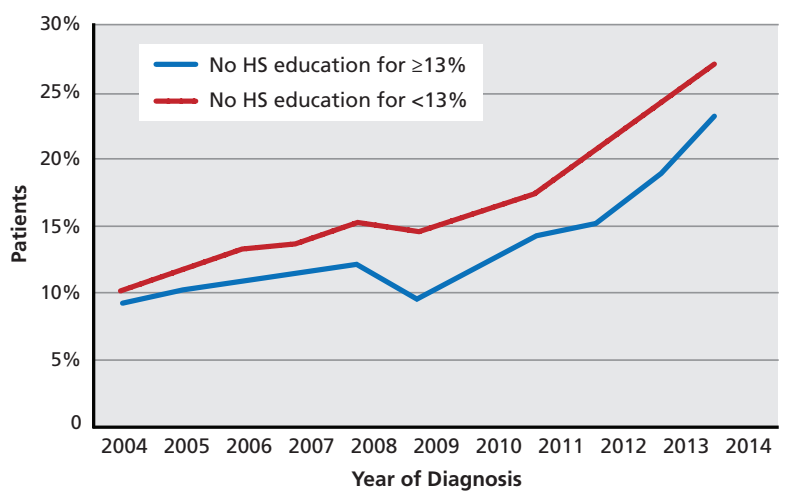

Figure 2. Brain stereotactic radiosurgery (SRS) utilization trends for patients with metastatic non-small cell lung cancer, breast cancer, colorectal cancer, or melanoma by sociodemographic characteristics. (A) Brain SRS utilization by median income, which represents the median household income in the patient's zip code. (B) Brain SRS utilization by insurance type. (C) Brain SRS utilization by reporting facility affiliation. (D) Brain SRS utilization by high school (HS) education, with HS education representing percentage of the adult population without a HS degree in the patient's zip code.

within Medicare-eligible patients that showed an increase in SRS use to $8.2 \%$ by $2005 .{ }^{9}$ Although direct comparison is not possible given substantial differences in the patient populations, our study is in line with these results, with an SRS use rate of $11.1 \%$ in the NCDB NSCLC subgroup in 2005. Our results suggest that the trend of increasing SRS use has not only continued but also accelerated during the most recent years.

Management of brain metastases is evolving, and the specific reasons for the increasing use of brain SRS remain unclear. RT recommendations for brain metastases vary widely and are dependent on the patient's performance status (PS) and extent of systemic and intracranial disease. Even with these clinical parameters defined, national guidelines leave ample room for clinician preference in using SRS and/or conventional WBRT in most clinical situations. ${ }^{6,15}$ Over the past decade, findings from several clinical trials have supported the use of SRS for brain metastases. Results of RTOG 9508, published during the first year of our study period, showed improved survival in patients with a single intracranial metastasis receiving brain SRS after WBRT and improved local control, PS, and steroid requirement for patients with up to 3 lesions. ${ }^{7}$ Three randomized trials published during the early-to-mid part of our study period suggested that SRS alone, without WBRT, had equivalent survival and was associated with relatively improved neurocognitive outcomes. ${ }^{8,10,11}$ The timing of publication of these studies coincides with an observed increase in the uptake of SRS. During our study period, multiple institutional SRS series were published, demonstrating promising efficacy for up to, and beyond, 10 intracranial lesions, ${ }^{20-22}$ in elderly patients, ${ }^{23}$ and in the postoperative setting, ${ }^{24}$ indicating an increased practitioner comfort level with brain SRS for a number of clinical situations. Additionally, further refinement of SRS technology occurred during our study period, including adoption of linear accelerator-based SRS ${ }^{25}$ and other frame- 


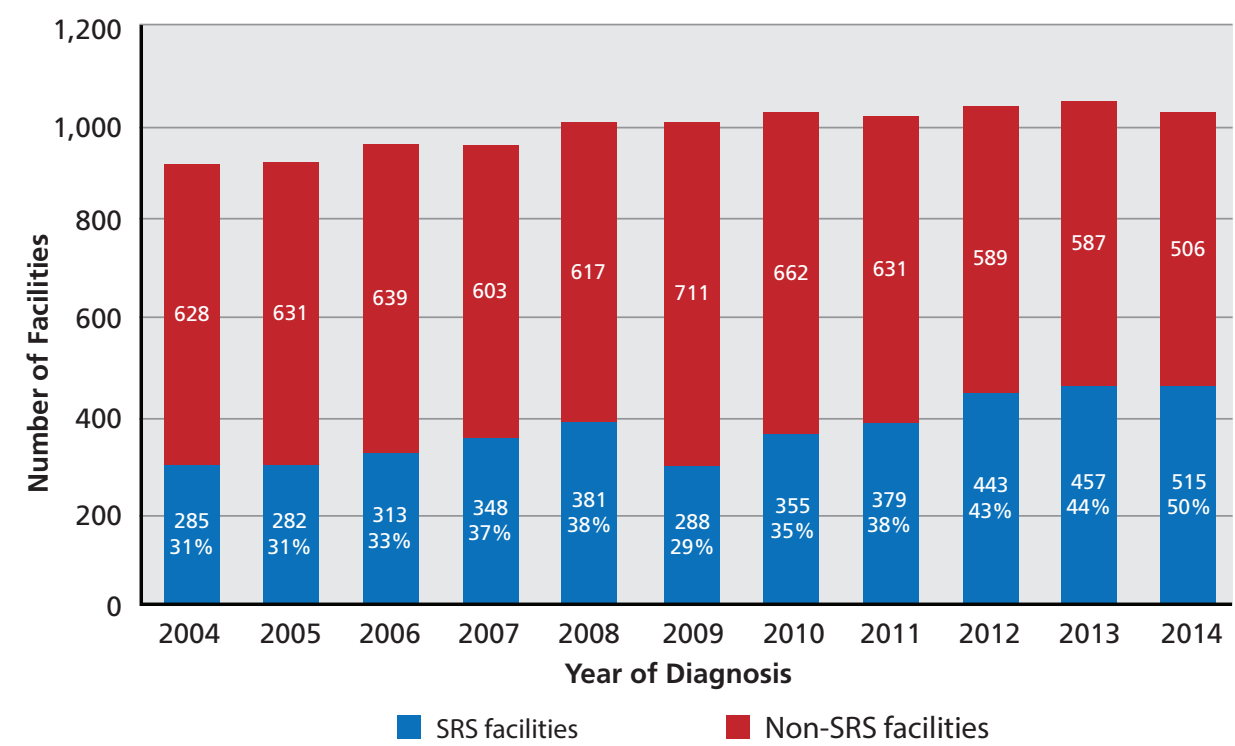

Figure 3. Number and proportion of facilities using brain stereotactic radiosurgery (SRS) from 2004 to 2014. Facilities captured are those that reported to the National Cancer Data Base during the diagnosis year; SRS facilities reported at least 1 case of brain SRS during the diagnosis year.

less SRS systems, ${ }^{26}$ which may have contributed to increased uptake.

Although SRS use increased substantially during the study period, it continues to be used in a low percentage of patients receiving brain RT overall. Whether the low overall rate is appropriate based on evidence-driven recommendations is unclear. Low overall SRS use may be due to clinical considerations, such as the presence of a high number of brain lesions, poor expected patient survival, diminished regional brain control, and lack of survival benefit compared with WBRT in randomized trials. Additionally, a recent secondary analysis of the JROSG 99-1 randomized trial suggested that patients with good prognoses may benefit from use of WBRT upfront followed by SRS compared with SRS alone. ${ }^{27}$ However, the low use rate may also reflect institutional biases, barriers to patient access, and cost concerns. A recent multi-institutional study suggested that the availability of on-site SRS was the most predictive factor for its use. ${ }^{28}$ The study indicates that access to SRS-equipped institutions may remain a barrier to SRS receipt, given that $50 \%$ of facilities in 2014 did not report a single patient who received brain SRS.

Additionally, our findings demonstrate differences in SRS uptake across socioeconomic groups. $\mathrm{Pa}$ tients from less wealthy and less educated areas and with Medicaid or lack of insurance were less likely to receive SRS, and this gap widened during the study period. Disparities across racial and ethnic groups are also seen, with black and Hispanic patients less likely to receive SRS overall, although uptake across race and ethnicity subgroups was equivalent during the study period. Much of the increase in SRS use appears to be driven by patients treated at academic facilities, which may have greater resources and multidisciplinary expertise necessary for directing an SRS program. Of note, we were not able to account for extent of intracranial or extracranial disease or PS,

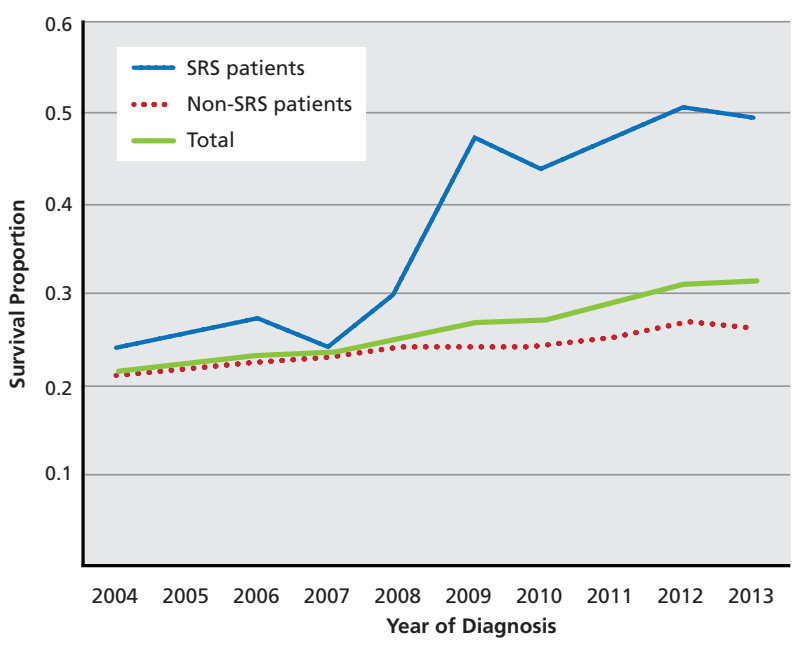

Figure 4. One-year actuarial overall survival from 2004 to 2013 by brain radiotherapy modality.

Abbreviation: SRS, stereotactic radiosurgery. 
which may confound these results, because it is possible that patients from lower socioeconomic backgrounds and with inferior insurance present with a greater burden of disease that precludes them from receiving brain SRS. It would be less likely, however, that this phenomenon would explain the growing disparity within these groups during the study period.

A diagnosis of melanoma was most highly associated with receipt of SRS, followed by CRC, NSCLC, and breast cancer. Rate of SRS use also increased most rapidly in melanoma, followed by CRC, NSCLC, and breast cancer. The association between SRS and melanoma is likely in part due to a general belief that SRS can be effective in treating radioresistant histologies, ${ }^{13}$ and to the successful incorporation of immunotherapy in the treatment of metastatic melanoma in the past 2 decades. Interestingly, CRC saw a dramatic increase in SRS use in the final year of the study period, although given the low number of patients with CRC overall, it is difficult to determine whether this is a statistical anomaly or indicates an actual trend.

This study showed that survival for patients receiving brain RT for metastatic disease is improving, and that this improvement is largely observed in patients selected to receive SRS. Although survival for SRS and non-SRS patients was similar in the early years of the study period, the discrepancy increased substantially in subsequent years.

The observed survival improvement during the study period is likely multifactorial. Substantial improvements in systemic therapy for metastatic disease have occurred during the past decade, including the advent of targeted agents and immunotherapy, which is likely improving survival in these patients. Given these improvements, it is plausible that more patients are becoming eligible for SRS. ${ }^{29,30}$ It is also possible that intracranial control has become more important in prolonging survival. ${ }^{27,31}$ Whether the increase in SRS use itself is causative for improved survival cannot be deduced from this study, and given the significant selection biases between the treatment cohorts and lack of potential confounder variables in the NCDB, we did not attempt such an analysis.

There are several additional limitations to this study. The NCDB captures only first-course treatment of particular diagnoses, and thus we cannot exclude the possibility that patients receiving SRS later went on to receive WBRT, or that patients in the non-SRS cohort went on to receive SRS later in their disease course. This study is only able to assess the upfront treatment strategy for patients. Despite this limitation, we believe the study adds valuable information in assessing the overall landscape of SRS use in the current management of brain metastases. The NCDB also lacks information regarding number of brain metastases, an important factor in the consideration of brain SRS use.

Although SRS is likely better accepted and more widely used in patients with $\leq 3$ brain metastases, the NCCN Clinical Practice Guidelines in Oncology for Central Nervous System Cancers ${ }^{6}$ now list SRS as an option for select patients with $>3$ brain metastases who have low tumor volume and good PS. There are several other important variables lacking in the NCDB that may confound the associations observed in our MVA regression analysis, including PS, extent of intracranial and extracranial disease, neurologic symptoms, and systemic therapy details, and we cannot control for this possibility. Additionally, although the NCDB captures approximately $70 \%$ of new cancer diagnoses, a recent study has suggested that, compared with population-based registries, Hispanic patients and certain geographic regions and diagnoses may be underrepresented within the NCDB, which may limit the generalizability of our results to the entire US population. ${ }^{32}$ However, the $\mathrm{NCDB}$ has the benefit of specific radiation site, modality, dose, and fractionation information, as well as the ability to conduct facility-level analyses, making it a particularly well-suited source for this study.

\section{Conclusions}

This national database analysis demonstrates steadily increasing — although modest overall-use and adoption of brain SRS in the United States during the past decade. The study identifies progressively widening sociodemographic disparities in the adoption of SRS, likely related to lack of access to this important technique. Further research is needed to determine the reasons for these worsening disparities and their clinical implications on intracranial control, neurocognitive toxicities, quality of life, and survival for patients with brain metastases. 
Kann et al

\section{References}

1. Barnholtz-Sloan JS, Sloan AE, Davis FG, et al. Incidence proportions of brain metastases in patients diagnosed (1973 to 2001) in the Metropolitan Detroit Cancer Surveillance System. J Clin Oncol 2004;22:2865-2872.

2. Nieder C, Spanne O, Mehta MP, et al. Presentation, patterns of care, and survival in patients with brain metastases. Cancer 2011;117:2505-2512.

3. Tabouret E, Chinot O, Metellus $P$, et al. Recent trends in epidemiology of brain metastases: an overview. Anticancer Res 2012;32:4655-4662.

4. Auchter RM, Lamond JP, Alexander I, et al. A multiinstitutional outcome and prognostic factor analysis of radiosurgery for resectable single brain metastasis. Int J Radiat Oncol Biol Phys 1996;35:27-35.

5. Shaw E, Scott C, Souhami L, et al. Single dose radiosurgical treatment of recurrent previously irradiated primary brain tumors and brain metastases: final report of RTOG protocol 90-05. Int J Radiat Oncol Biol Phys 2000;47:291-298.

6. Nabors LB, Portnow J, Ammirati M, et al. NCCN Clinical Practice Guidelines in Oncology: Central Nervous System Cancers. Version 1.2016. Accessed May 31, 2017. To view the most recent version of these guidelines, visit NCCN.org.

7. Andrews DW, Scott CB, Sperduto PW, et al. Whole brain radiation therapy with or without stereotactic radiosurgery boost for patients with one to three brain metastases: phase III results of the RTOG 9508 randomised trial. Lancet 2004;363:1665-1672

8. Aoyama $\mathrm{H}$, Shirato $\mathrm{H}$, Tago $\mathrm{M}$, et al. Stereotactic radiosurgery plus wholebrain radiation therapy vs stereotactic radiosurgery alone for treatment of brain metastases: a randomized controlled trial. JAMA 2006;295:24832491.

9. Halasz LM, Weeks JC, Neville BA, et al. Use of stereotactic radiosurgery for brain metastases from non-small cell lung cancer in the United States. Int J Radiat Oncol Biol Phys 2013;85:e109-116.

10. Kocher M, Soffietti R, Abacioglu U, et al. Adjuvant whole-brain radiotherapy versus observation after radiosurgery or surgical resection of one to three cerebral metastases: results of the EORTC 22952-26001 study. J Clin Oncol 2011;29:134-141.

11. Chang EL, Wefel JS, Hess KR, et al. Neurocognition in patients with brain metastases treated with radiosurgery or radiosurgery plus whole-brain irradiation: a randomised controlled trial. Lancet Oncol 2009;10:10371044.

12. Tsao M, Xu W, Sahgal A. A meta-analysis evaluating stereotactic radiosurgery, whole-brain radiotherapy, or both for patients presenting with a limited number of brain metastases. Cancer 2012;118:2486-2493.

13. Chang EL, Selek U, Hassenbusch SJ, et al. Outcome variation among "radioresistant" brain metastases treated with stereotactic radiosurgery. Neurosurgery 2005;56:936-945.

14. Sperduto PW, Chao ST, Sneed PK, et al. Diagnosis-specific prognostic factors, indexes, and treatment outcomes for patients with newly diagnosed brain metastases: a multi-institutional analysis of 4,259 patients. Int J Radiat Oncol Biol Phys 2010;77:655-661.

15. Tsao MN, Rades D, Wirth A, et al. Radiotherapeutic and surgical management for newly diagnosed brain metastasis(es): an American Society for Radiation Oncology evidence-based guideline. Pract Radiat Oncol 2012;2:210-225.

16. Sandler KA, Shaverdian N, Cook RR, et al. Treatment trends for patients with brain metastases: does practice reflect the data? Cancer 2017;123:2274-2282.
17. American College of Surgeons. National Cancer Data Base: Welcome to the 2015 PUF Data Dictionary. Available at: http://ncdbpuf.facs.org. Accessed November 20, 2017.

18. Paternoster R, Brame $R$, Mazerolle P, Piquero A. Using the correct statistical test for the equality of regression coefficients research note. Criminology 1998;36:859-866.

19. Wilcox RR. Comparing the slopes of two independent regression lines when there is complete heteroscedasticity. $\mathrm{Br} \mathrm{J}$ Math Stat Psychol 1997;50:309-317.

20. Grandhi R, Kondziolka D, Panczykowski D, et al. Stereotactic radiosurgery using the Leksell Gamma Knife Perfexion unit in the management of patients with 10 or more brain metastases. J Neurosurg 2012;117:237-245.

21. Yamamoto M, Serizawa $T$, Shuto $T$, et al. Stereotactic radiosurgery for patients with multiple brain metastases (JLGK0901): a multi-institutional prospective observational study. Lancet Oncol 2014;15:387-395.

22. Yamamoto $M$, Kawabe $T$, Sato $Y$, et al. Stereotactic radiosurgery for patients with multiple brain metastases: a case-matched study comparing treatment results for patients with $2-9$ versus 10 or more tumors. Neurosurg 2014;121(Suppl):16-25.

23. Yomo $S$, Hayashi M. Is upfront stereotactic radiosurgery a rational treatment option for very elderly patients with brain metastases? A retrospective analysis of 106 consecutive patients age 80 years and older. BMC Cancer 2016;16:948.

24. Brennan $C$, Yang TJ, Hilden $P$, et al. A phase 2 trial of stereotactic radiosurgery boost after surgical resection for brain metastases. Int J Radiat Oncol Biol Phys 2014;88:130-136.

25. Park HS, Wang EH, Rutter CE, et al. Changing practice patterns of gamma knife versus linear accelerator-based stereotactic radiosurgery for brain metastases in the US. J Neurosurg 2016;124:1018-1024.

26. Rodrigues G, Yartsev S, Yaremko B, et al. Phase I trial of simultaneous infield boost with helical tomotherapy for patients with one to three brain metastases. Int J Radiat Oncol Biol Phys 2011;80:1128-1133.

27. Aoyama $H$, Tago $M$, Shirato $H$. stereotactic radiosurgery with or without whole-brain radiotherapy for brain metastases: secondary analysis of the JROSG 99-1 randomized clinical trial. JAMA Oncol 2015;1:457-464.

28. Hodgson DC, Charpentier AM, Cigsar C, et al. A multi-institutional study of factors influencing the use of stereotactic radiosurgery for brain metastases. Int J Radiat Oncol Biol Phys 2013;85:335-340.

29. Kiess AP, Wolchok JD, Barker CA, et al. Stereotactic radiosurgery for melanoma brain metastases in patients receiving ipilimumab: safety profile and efficacy of combined treatment. Int J Radiat Oncol Biol Phys 2015;92:368-375.

30. Knisely JPS, Yu JB, Flanigan J, et al. Radiosurgery for melanoma brain metastases in the ipilimumab era and the possibility of longer survival. J Neurosurg 2012;117:227-233.

31. Magnuson WJ, Lester-Coll NH, Wu AJ, et al. Management of brain metastases in tyrosine kinase inhibitor-naïve epidermal growth factor receptor-mutant non-small-cell lung cancer: a retrospective multiinstitutional analysis. J Clin Oncol 2017;35:1070-1077.

32. Halasz LM, Uno $H$, Hughes $M$, et al. Comparative effectiveness of stereotactic radiosurgery versus whole-brain radiation therapy for patients with brain metastases from breast or non-small cell lung cancer. Cancer 2016;122:2091-2100. 